\title{
On some nonlinear boundary value problems related to a Black-Scholes model with transaction costs
}

\author{
Rubén Figueroa ${ }^{\text {* }}$ and Maria do Rosário Grossinho²
}

\section{"Correspondence:}

ruben.figueroa@usc.es

'Department of Mathematical

Analysis, University of Santiago de

Compostela, Campus Vida, Santiago

de Compostela, 15782, Spain

Full list of author information is

available at the end of the article

\begin{abstract}
We deal with some generalizations on a Black-Scholes model arising in financial mathematics. As a novelty in this paper, we consider a variable volatility and abstract functional boundary conditions, which allow us to treat a very large class of problems involving Black-Scholes equation. Our main results involve the existence of extremal solutions in presence of lower and upper solutions. Some examples of applications are provided too.
\end{abstract}

Keywords: Black-Scholes equation; functional conditions; discontinuous ODE's

\section{Introduction}

In this paper we are concerned with the following nonlinear boundary value problem:

$$
\left\{\begin{array}{l}
x^{3}\left(V^{\prime \prime}\right)^{2}(x)+p(x) x^{2} V^{\prime \prime}(x)+q(x)\left(x V^{\prime}(x)-V(x)\right)=0, \\
B_{1}(V(c), V)=0, \quad B_{2}(V(d), V)=0,
\end{array}\right.
$$

where $p, q$ are nonnegative bounded functions which could be discontinuous in $[c, d]$, $c, d>0$, and $B_{i}: \mathbb{R} \times \mathcal{C}([c, d]) \longrightarrow \mathbb{R}, i=1,2$, are functions which satisfy some conditions that we will state later. We observe that under this framework, a large class of boundary conditions is included, namely:

(1) Dirichlet conditions: $B_{1}(V(c), V)=V(c)-V_{c}, B_{2}(V(d), V)=V(d)-V_{d}$;

(2) Initial-integral conditions: $B_{1}(V(c), V)=V(c)-\int_{c}^{d} k(x) V(x) d x$;

(3) Multipoint conditions: $B_{2}(V(d), V)=V(d)-\sum_{j=1}^{n} V\left(x_{j}\right)$, with $V_{c}, V_{d} \in \mathbb{R}$.

This study follows and generalizes the results contained in [1] with respect to the problem

$$
\left\{\begin{array}{l}
x^{3}\left(V^{\prime \prime}\right)^{2}(x)+p x^{2} V^{\prime \prime}(x)+q\left(x V^{\prime}(x)-V(x)\right)=0 \\
V(c)=V_{c}, \quad V(d)=V_{d}
\end{array}\right.
$$

In [1], it is assumed that $p, q$ are positive constants and $V_{c}<V_{d}$. The contributions of the present paper are the following. First we address this problem but drop the condition $V_{c}<V_{d}$, and replace the constants $p, q$ by two functions $p(x), q(x)$; second, we replace

(c) 2015 Figueroa and Grossinho. This article is distributed under the terms of the Creative Commons Attribution 4.0 International License (http://creativecommons.org/licenses/by/4.0/), which permits unrestricted use, distribution, and reproduction in any medium, provided you give appropriate credit to the original author(s) and the source, provide a link to the Creative Commons license, and indicate if changes were made. 
the Dirichlet conditions by functional boundary conditions, which allows us to consider a very large class of problems for the equation of (2).

These problems are related to financial option pricing since they address the existence of stationary solutions of a class of generalizations of the classical Black-Scholes model (BS), introduced in 1973 [2], with equation

$$
\frac{\partial V}{\partial t}+\frac{1}{2} \sigma^{2} S^{2} \frac{\partial^{2} V}{\partial S^{2}}+r\left(S \frac{\partial V}{\partial S}-V\right)=0
$$

where $V$ represents the value of a call or put option, depending on an underlying asset $S$ and on time $t, r$ is the interest short rate and $\sigma$ is the volatility of the asset price. In the (BS) model, $S$ is modeled as a geometric Brownian motion, and no costs are considered when financial transactions hold.

Suppose that transaction costs are included in the model under the assumption that they are a percentage of the transaction, given as in [3] by a linear function $h$ of the number of shares traded, i.e., $h(\eta)=a-b \eta$, where $\eta$ is the number of shares traded and $a, b>0$. The following nonlinear BS type equation is obtained, where $\Delta t$ is the interval between transactions,

$$
V_{t}+\frac{1}{2} \sigma^{2} S^{2} V_{S S}-a \sigma S^{2} \sqrt{\frac{2}{\pi \Delta t}}\left|V_{S S}\right|+b S^{3} \sigma^{2} V_{S S}^{2}+r\left(S V_{S}-V\right)=0
$$

Then, if $a$ is small enough and $V_{S S}>0$ (see $[1,3,4]$ ), the following nonlinear version of (3) is obtained:

$$
\frac{\partial V}{\partial t}+\frac{1}{2} \tilde{\sigma}^{2} S^{2} \frac{\partial^{2} V}{\partial S^{2}}+b \sigma^{2} S^{3}\left(\frac{\partial^{2} V}{\partial S^{2}}\right)^{2}+r\left(S \frac{\partial V}{\partial S}-V\right)=0,
$$

where $\tilde{\sigma}=\sigma^{2}\left(1-2 \frac{a}{\sigma} \sqrt{\frac{2}{\pi \Delta t}}\right)>0$ and is commonly denoted by adjusted volatility. Now, if we consider the stationary version of (4), we obtain the above ordinary differential equation (2) where $p=\frac{\tilde{\sigma}^{2}}{2 b \sigma^{2}}$ and $q=\frac{r}{b \sigma^{2}}$ are constants.

This paper is organized as follows. In Section 2, we introduce an auxiliary nonlinear boundary value problem and the notions of upper and lower solutions used later. In Section 3, we consider problem (1) with Dirichlet boundary conditions. So, we start from paper [1] and we generalize it by considering $p(x), q(x)$ instead of constants $p, q$, which corresponds to variable volatility in the (BS) model, and by dropping the condition $V_{c}<V_{d}$. In Section 4, we deal with problem (1) on its full version, that is, with functional boundary conditions. Namely, we provide a result on the existence of extremal solutions between lower and upper solutions by using a generalized iteration with Dirichlet problems. Some examples of application are provided, too.

\section{Auxiliary problem and upper and lower solutions}

If we look to the equation of (1)

$$
x^{3}\left(V^{\prime \prime}\right)^{2}(x)+p(x) x^{2} V^{\prime \prime}(x)+q(x)\left(x V^{\prime}(x)-V(x)\right)=0
$$


and, as in $[3,4]$ and [1], solve it algebraically in order to $V^{\prime \prime}$, we obtain

$$
V^{\prime \prime}(x)=-\frac{p(x) x^{2} \pm \sqrt{p^{2}(x) x^{4}-4 x^{3} q(x)\left(x V^{\prime}(x)-V(x)\right)}}{2 x^{3}} .
$$

Since we are interested in convex solutions of (1), they can exist only if

$$
V^{\prime \prime}(x)=-\frac{p(x) x^{2}+\sqrt{p^{2}(x) x^{4}-4 x^{3} q(x)\left(x V^{\prime}(x)-V(x)\right)}}{2 x^{3}},
$$

and

$$
-\left(x V^{\prime}(x)-V(x)\right) \geq 0 .
$$

These facts lead us to consider first the following related problem:

$$
\left\{\begin{array}{l}
V^{\prime \prime}(x)+H\left(x, V(x), V^{\prime}(x)\right)=0, \\
B_{1}(V(c), V)=0, \quad B_{2}(V(d), V)=0,
\end{array}\right.
$$

where

$$
H(x, y, z)=\frac{p(x) x^{2}-\sqrt{p^{2}(x) x^{4}+4 x^{3} q(x)|x z-y|}}{2 x^{3}} .
$$

Then we will see how solutions of this problem are solutions of our original problem.

We will use the method of upper and lower solutions for this problem and we will begin by considering the classical notions of $\mathcal{C}^{2}$-lower and upper solutions. However, afterwards, we will use some weaker notions, namely we will need a notion that allows lower solutions to have 'angles'. In fact, we will consider a maximum of two classical $\mathcal{C}^{2}$-lower solutions which is not necessarily differentiable and can exhibit an 'angle'. So, denoting by $D_{-} f(x)$ and $D^{+} f(x)$, respectively, the lower left-hand and the upper right-hand Dini-derivatives of the function $f$ at $x$, we introduce the following definitions (see [5]).

Definition 2.1 We say that $\alpha \in A C([c, d])$ is a lower solution for problem (8) if

$$
B_{1}(\alpha(c), \alpha) \leq 0, \quad B_{2}(\alpha(d), \alpha) \leq 0,
$$

and for each $x_{0} \in(c, d)$ one of the following conditions holds:

(1) $D_{-} \alpha\left(x_{0}\right)<D^{+} \alpha\left(x_{0}\right)$;

(2) There exists an open interval $J_{0}$ such that $x_{0} \in J_{0} \subset(c, d), \alpha_{J_{0}} \in W^{2,1}\left(J_{0}\right)$, and for almost all $x \in J_{0}$ we have

$$
\alpha^{\prime \prime}(x)+H\left(x, \alpha(x), \alpha^{\prime}(x)\right) \geq 0 .
$$

We say that $\beta \in A C([c, d])$ is an upper solution for problem (8) if

$$
B_{1}(\beta(c), \beta) \geq 0, \quad B_{2}(\beta(d), \beta) \geq 0,
$$

and for each $x_{0} \in(c, d)$ one of the following conditions holds:

(1) $D^{-} \beta\left(x_{0}\right)>D_{+} \beta\left(x_{0}\right)$; 
(2) There exists an open interval $J_{0}$ such that $x_{0} \in J_{0} \subset(c, d), \beta_{\mid J_{0}} \in W^{2,1}\left(J_{0}\right)$, and for almost all $x \in J_{0}$ we have

$$
\beta^{\prime \prime}(x)+H\left(x, \beta(x), \beta^{\prime}(x)\right) \leq 0
$$

Notice that if $\alpha, \beta$ are classical $\mathcal{C}^{2}$-lower and upper solutions for problem (8), then they are also lower and upper solutions in the sense referred above.

\section{Nonlinear problem with Dirichlet conditions}

Consider problem (1) with

$$
B_{1}(V(c), V)=V(c)-V_{c}, \quad B_{2}(V(d), V)=V(d)-V_{d},
$$

that is, with standard Dirichlet conditions. So, we have in this case

$$
\left\{\begin{array}{l}
x^{3}\left(V^{\prime \prime}\right)^{2}(x)+p(x) x^{2} V^{\prime \prime}(x)+q(x)\left(x V^{\prime}(x)-V(x)\right)=0, \\
V(c)=V_{c}, \quad V(d)=V_{d} .
\end{array}\right.
$$

The auxiliary problem referred in the previous sections is now

$$
\left\{\begin{array}{l}
V^{\prime \prime}(x)+H\left(x, V(x), V^{\prime}(x)\right)=0 \\
V(c)=V_{c}, \quad V(d)=V_{d}
\end{array}\right.
$$

where the function $H$ is given by (9).

From the study of problem (11), we will deduce later existence and localization results for problem (10). Next proposition establishes adequately the existence of classical $\mathcal{C}^{2}$-upper and lower solutions for (11).

Proposition 3.1 The following assertions hold:

(1) If $\frac{V_{d}}{d} \leq \frac{V_{c}}{c}$, then the function

$$
\alpha_{1}(x)=\frac{V_{d}}{d} x
$$

is a $\mathcal{C}^{2}$-lower solution for problem (11).

The converse is also valid.

(2) Take $k>0$ such that

$$
k \geq \sqrt{\frac{Q}{c^{3}}} \sqrt{\max _{x \in[c, d]}\left|\frac{k}{2}\left(x^{2}-c d\right)+\frac{c V_{d}-d V_{c}}{d-c}\right|},
$$

where

$$
Q=\max _{x \in[c, d]} q(x) .
$$

Then the function

$$
\alpha_{k}(x)=\frac{k}{2} x^{2}+\left(\frac{V_{d}-V_{c}}{d-c}-\frac{k}{2}(d+c)\right) x+\frac{k}{2} c d-\frac{c V_{d}-d V_{c}}{d-c}
$$

is a $\mathcal{C}^{2}$-lower solution for problem (11). 
(3) The function

$$
\beta(x)=\frac{V_{d}-V_{c}}{d-c} x+\frac{d V_{c}-c V_{d}}{d-c}, \quad x \in[c, d]
$$

is a $\mathcal{C}^{2}$-upper solution for problem (11).

Proof (1) If $\frac{V_{d}}{d} \leq \frac{V_{c}}{c}$, the thesis follows since

$$
\alpha_{1}(c)=\frac{V_{d}}{d} c \leq V_{c}, \quad \alpha_{1}(d)=V_{d}
$$

and, in $(c, d)$,

$$
\alpha_{1}^{\prime \prime}(x)+H\left(x, \alpha_{1}(x), \alpha_{1}^{\prime}(x)\right)=0 .
$$

Conversely, if $\alpha_{1}$ is a lower solution for problem (11), then $\alpha_{1}(c)=\frac{V_{d}}{d} c \leq V_{c}$, which implies $\frac{V_{d}}{d} \leq \frac{V_{c}}{c}$.

(2) Observe that $\alpha_{k}(c)=V_{c}, \alpha_{k}(d)=V_{d}$. On the other hand, as

$$
\begin{aligned}
H\left(x, \alpha_{k}(x), \alpha_{k}^{\prime}(x)\right) & \geq \frac{p(x) x^{2}-\sqrt{p^{2}(x) x^{4}}-\sqrt{4 x^{3} q(x)\left|x \alpha_{k}^{\prime}(x)-\alpha_{k}(x)\right|}}{2 x^{3}} \\
& \geq-\sqrt{\frac{q(x)}{x^{3}}} \sqrt{\left|x \alpha_{2}^{\prime}(x)-\alpha_{k}(x)\right|}, \\
& \geq-\sqrt{\frac{Q}{c^{3}} \sqrt{\max _{x \in[c, d]}\left|\frac{k}{2}\left(x^{2}-c d\right)+\frac{V_{d} c-V_{c} d}{d-c}\right|},}
\end{aligned}
$$

then

$$
\alpha_{k}^{\prime \prime}(x)+H\left(x, \alpha_{k}(x), \alpha_{k}^{\prime}(x)\right) \geq k-\sqrt{\frac{Q}{c^{3}}} \sqrt{\max _{x \in[c, d]}\left|\frac{k}{2}\left(x^{2}-c d\right)-V_{c}+\frac{V_{d}-V_{c}}{d-c} c\right|} .
$$

Since $k>0$ satisfies hypothesis (13), we derive

$$
\alpha_{k}^{\prime \prime}(x)+H\left(x, \alpha_{k}(x), \alpha_{k}^{\prime}(x)\right) \geq 0 .
$$

Then the assertion holds.

(3) The thesis follows easily from the fact that

$$
\beta^{\prime \prime}(x)+H\left(x, \beta(x), \beta^{\prime}(x)\right)=H\left(x, \beta(x), \beta^{\prime}(x)\right) \leq 0
$$

and

$$
\beta(c)=V_{c}, \quad \beta(d)=V_{d}
$$

Remark 3.1 (1) Notice that there is no ambiguity in considering $k$ big enough such that condition (13) holds. In fact, it is easy to see that the maximum in (13) depends on $k$ and 
is attained in the following way:

$$
\begin{aligned}
& \max _{x \in[c, d]}\left|\frac{k}{2}\left(x^{2}-c d\right)+\frac{V_{d} c-V_{c} d}{d-c}\right| \\
& =\max \left\{\left|\frac{k}{2}\left(d^{2}-c d\right)+\frac{V_{d} c-V_{c} d}{d-c}\right|,\left|\frac{k}{2}\left(c^{2}-c d\right)+\frac{V_{d} c-V_{c} d}{d-c}\right|\right\} .
\end{aligned}
$$

So, it is clear that in the proof of the previous proposition, we could choose $k$ satisfying (13) since

$$
\lim _{k \rightarrow \infty} \frac{k}{\sqrt{k}}=+\infty
$$

(2) Observe also that the lower solution $\alpha_{k}$ can be written as $\alpha_{k}(x)=\beta(x)+\theta(x)$, where

$$
\theta(x)=\frac{k}{2}\left(x^{2}-(d+c) x+c d\right)
$$

In fact,

$$
\begin{aligned}
\alpha_{k}(x) & =\frac{k}{2} x^{2}+\left(\frac{V_{d}-V_{c}}{d-c}-\frac{k}{2}(d+c)\right) x+\frac{k}{2} c d-\frac{V_{d} c-V_{c} d}{d-c} \\
& =\left(\frac{V_{d}-V_{c}}{d-c}\right) x+\frac{d V_{c}-c V_{d}}{d-c}+\frac{k}{2}\left(x^{2}-(d+c) x+c d\right) .
\end{aligned}
$$

The function $\theta$ is quadratic, vanishes at $x=c$ and $x=d$, and is negative in $] c, d[$.

Notation 3.1 Given two functions $\phi \leq \psi$ in $[c, d]$, let us denote by $[\phi, \psi]$ the functional interval

$$
[\phi, \psi]=\left\{V \in W^{2,1}([c, d]): \phi(x) \leq V(x) \leq \psi(x) \text { for all } x \in[c, d]\right\}
$$

Next, we state an existence and localization result for problem (11).

Theorem 3.1 Let $\alpha_{1}, \alpha_{k}$ and $\beta$ be the functions defined in the previous proposition.

(a) If $\frac{V_{d}}{d} \leq \frac{V_{c}}{c}$, then problem (11) has extremal $W^{2,1}$-solutions, that is, the least and the greatest one, in the functional interval $\left[\alpha_{1}, \beta\right]$.

(b) If $k>0$ satisfies (13), then problem (11) has extremal $W^{2,1}$-solutions in the functional interval $\left[\alpha_{k}, \beta\right]$.

Proof Consider in case (a)

$$
E_{1}=\left\{(x, y, z) \in[c, d] \times \mathbb{R}^{2}: \alpha_{1}(x) \leq y \leq \beta(x)\right\},
$$

and in case (b)

$$
E_{2}=\left\{(x, y, z) \in[c, d] \times \mathbb{R}^{2}: \alpha_{k}(x) \leq y \leq \beta(x)\right\} .
$$


It is clear that $H$ is continuous in $E_{1}$ and $E_{2}$. Moreover, we have that

$$
|H(x, y, z)| \leq \frac{p(x)}{x}+\sqrt{\frac{q(x)}{x^{3}} \beta(x)}+\frac{\sqrt{q(x)}}{x} \sqrt{|z|}
$$

for all $(x, y, z) \in E_{1}$ or $(x, y, z) \in E_{2}$. Then, putting

$$
\hat{A}=\max _{x \in[c, d]}\left(\frac{p(x)}{x}+\sqrt{\frac{q(x)}{x^{3}} \beta(x)}\right), \quad \hat{B}=\max _{x \in[c, d]} \frac{\sqrt{q(x)}}{x}
$$

we derive that

$$
|H(x, y, z)| \leq \hat{A}+\hat{B} \sqrt{|z|}
$$

for $(x, y, z) \in E_{1}$ or $(x, y, z) \in E_{2}$, respectively. This inequality guarantees that the function $H$ satisfies the (classical) Nagumo condition both in $E_{1}$ and $E_{2}$. Using the fact that $\alpha_{1}, \alpha_{k}$ are $\mathcal{C}^{2}$-lower solutions in cases (a) and (b), respectively, and $\beta$ is a $\mathcal{C}^{2}$-upper solution for problem (11) such that

$$
\alpha_{1} \leq \beta, \quad \alpha_{k} \leq \beta,
$$

the conclusion holds by application of a well-known result contained in [6].

Corollary 3.1 Let $\frac{V_{d}}{d} \leq \frac{V_{c}}{c}$ and $k>0$ satisfy (13) and consider the functions $\alpha_{1}, \alpha_{k}$ and $\beta$ defined, respectively, by (12), (14), (15). Define

$$
\alpha(x)=\max \left\{\alpha_{1}(x), \alpha_{k}(x)\right\} .
$$

Then problem (11) has the extremal $W^{2,1}$-solutions in the interval

$$
[\alpha, \beta]=\left\{V \in W^{2,1}([c, d]): \alpha(x) \leq V(x) \leq \beta(x) \text { for all } x \in[c, d]\right\}
$$

Proof We observe that

$$
\alpha(x)=\max \left\{\alpha_{1}(x), \alpha_{k}(x)\right\}
$$

is a lower solution for problem (11) in the sense defined in the previous section (not necessarily $\mathcal{C}^{2}$ ) and, as before, $\beta$ is a $\mathcal{C}^{2}$-upper solution for problem (11). Similarly to the proof of Theorem 3.1, the Nagumo condition holds in

$$
E=\left\{(x, y, z) \in[c, d] \times \mathbb{R}^{2}: \alpha(x) \leq y \leq \beta(x)\right\} .
$$

Then the result follows from an existence theorem contained in [6].

Proposition 3.2 The following assertions hold:

(a) Every solution $V$ of problem (11) is convex. 
(b) Consider $\alpha_{1}$ defined by (12). Then every solution $V$ of problem (11) such that $V \geq \alpha_{1}$ satisfies, for all in $x \in[c, d]$,

$$
x V^{\prime}(x)-V(x) \leq 0
$$

Proof (a) Clearly, the convexity of solutions of (11) derives from the fact that

$$
\left.V^{\prime \prime}(x)=-H\left(x, V(x), V^{\prime}(x)\right) \geq 0 \quad \text { for all } x \in\right] c, d[
$$

and from the continuity in $c$ and $d$.

(b) Let $V$ be a solution of problem (11) such that $V \geq \alpha_{1}$ in $[c, d]$. We claim that $\frac{V_{d}}{d} \geq$ $V^{\prime}(d)$. In fact, $\alpha_{1}=\frac{V_{d}}{d} x \leq V(x)$ implies that $\frac{V_{d}}{d} \geq \frac{V(x)-V_{d}}{x-d}$ in $[c, d]$, and then, letting $x \rightarrow d$, we obtain $\frac{V_{d}}{d} \geq V^{\prime}(d)$. This inequality together with the fact that the function $x \in[c, d] \longmapsto$ $x V^{\prime}(x)-V(x)$ is nondecreasing implies that $x V^{\prime}(x)-V(x) \leq 0$ for all $x \in[c, d]$.

Next theorem establishes the relations between the convex solutions of (11) and of (10).

Theorem 3.2 Consider problems (11) and (10). Then

(a) If $\frac{V_{d}}{d} \leq \frac{V_{c}}{c}$, every solution $V$ of (11) provided by Theorem 3.1 is a convex solution of (10).

(b) Every convex solution $V$ of problem (10) is a convex solution of problem (11).

Proof As for (a), let $\frac{V_{d}}{d} \leq \frac{V_{c}}{c}$. Then $\alpha_{1}$ is a lower solution of (11) and every solution $V$ of (11) provided by Theorem 3.1 satisfies $V(x) \geq \alpha_{1}(x)$. Hence, by Proposition 3.2, $V$ is convex and

$$
\left|x V^{\prime}(x)-V(x)\right|=-\left(x V^{\prime}(x)-V(x)\right)
$$

So

$$
V^{\prime \prime}(x)=-H\left(x, V(x), V^{\prime}(x)\right)=\frac{-p(x) x^{2}+\sqrt{p(x) x^{4}-4 q(x) x^{3}\left(x V^{\prime}(x)-V(x)\right)}}{2 x^{3}},
$$

which shows clearly that $V$ is a convex function that solves (10).

As for (b), let $V$ be a convex solution of (10). Then

$$
x^{3}\left(V^{\prime \prime}\right)^{2}(x)+p(x) x^{2} V^{\prime \prime}(x)+q(x)\left(x V^{\prime}(x)-V(x)\right)=0,
$$

and as $V^{\prime \prime} \geq 0, p(x) \geq 0$ and $0<c \leq x \leq d$, then

$$
q(x)\left(x V^{\prime}(x)-V(x)\right) \leq 0 .
$$

Then

$$
x^{6}\left(V^{\prime \prime}\right)^{2}(x)+p(x) x^{5} V^{\prime \prime}(x)=x^{3} q(x)\left|x V^{\prime}(x)-V(x)\right|,
$$

that is, by adding $\frac{1}{4} p^{2}(x) x^{4}$ to both members,

$$
\left(x^{3} V^{\prime \prime}(x)+\frac{1}{2} p(x) x^{2}\right)^{2}=\frac{1}{4} p^{2}(x) x^{4}+x^{3} q(x)\left|x V^{\prime}(x)-V(x)\right| .
$$


Applying the square root, we obtain

$$
x^{3} V^{\prime \prime}(x)+\frac{1}{2} p(x) x^{2}=\sqrt{\frac{1}{4} p^{2}(x) x^{4}+x^{3} q(x) \mid x V^{\prime}(x)-V(x)} \mid
$$

and then

$$
V^{\prime \prime}(x)=\frac{-p(x) x^{2}+\sqrt{p^{2}(x) x^{4}+4 x^{3} q(x)\left|x V^{\prime}(x)-V(x)\right|}}{2 x^{3}}=-H\left(x, V(x), V^{\prime}(x)\right) .
$$

So, $V$ solves (10).

From Theorem 3.1, Theorem 3.2 and Corollary 3.1, it is clear that the following existence and localization result holds.

Theorem 3.3 Consider problem (1) with standard Dirichlet conditions, that is,

$$
\left\{\begin{array}{l}
x^{3}\left(V^{\prime \prime}\right)^{2}(x)+p(x) x^{2} V^{\prime \prime}(x)+q(x)\left(x V^{\prime}(x)-V(x)\right)=0, \\
V(c)=V_{c}, \quad V(d)=V_{d} .
\end{array}\right.
$$

(1) If $\frac{V_{d}}{d} \leq \frac{V_{c}}{c}$, then this problem has the extremal convex $W^{2,1}$-solutions in the functional interval $\left[\alpha_{1}, \beta\right]$, where $\alpha_{1}$ and $\beta$ are provided, respectively, by (12) and (15);

(2) If $\frac{V_{d}}{d} \leq \frac{V_{c}}{c}$ and $k>0$ satisfies (13), then this problem has the extremal convex $W^{2,1}$-solutions in the functional interval $[\alpha, \beta]$, where

$$
\alpha(x)=\max \left\{\alpha_{1}(x), \alpha_{k}(x)\right\}
$$

and $\alpha_{1}, \alpha_{k}, \beta$ are provided, respectively, by (12), (14), (15).

Remark 3.2 Under the hypotheses of the above theorem, observe that if $\frac{V_{d}}{d}=\frac{V_{c}}{c}$ then $\alpha_{1}$ is a solution of problem (10). On the other hand, in the periodic case, $V_{c}=V_{d}$, the constant function $V \equiv V_{c}$ is a solution of (10).

Example 3.1 Consider problem (10) in the interval $[c, d]=[2,6]$, with

$$
p(x)=1+x^{3}, \quad q(x)=[x],
$$

where [.] denotes integer part, and boundary conditions $V(2)=9, V(6)=1$. Notice that in this case it is $V_{c}>V_{d}$. From condition (13), $k>0$ must satisfy

$$
k \geq \sqrt{\frac{6}{8}} \sqrt{\max \{|-4 k-13|,|12 k-13|\}},
$$

so simple computations show that $k=8$ satisfies (13). Then the function

$$
\alpha(x)=4 x^{2}-34 x+61
$$

is a lower solution for this problem. At the same time,

$$
\beta(x)=13-2 x
$$

is an upper solution. 
Then, by application of Theorem 3.3, problem (10) with $p(x)=1+x^{3}, q(x)=[x]$, and boundary conditions $V(2)=9, V(6)=1$, has the extremal solutions in the functional interval

$$
\left[\max \left\{\frac{1}{6} x, 4 x^{2}-34 x+61\right\}, 13-2 x\right] .
$$

\section{Problem with functional boundary conditions}

In this section we deal with problem (8) on its full expression and, as said in the introduction, we will use a generalized monotone method. In the construction of this method we will use two technical lemmas. First of them is the following generalization of Bolzano's theorem.

Lemma 4.1 ([7], Lemma 2.3) Let $a, b \in \mathbb{R}, a \leq b$, and $h: \mathbb{R} \rightarrow \mathbb{R}$ be a function satisfying $h(a) \leq 0 \leq h(b)$ and

$$
\liminf _{z \rightarrow x^{-}} h(z) \geq h(x) \geq \limsup _{z \rightarrow x^{+}} h(z) \quad \text { for all } x \in[a, b]
$$

Then there exist $c_{1}, c_{2} \in[a, b]$ such that $h\left(c_{1}\right)=0=h\left(c_{2}\right)$ and if $h(c)=0$ for some $c \in[a, b]$, then $c_{1} \leq c \leq c_{2}$, that is, $c_{1}$ and $c_{2}$ are, respectively, the least zero and the greatest one of $h$ in $[a, b]$.

The second auxiliary result we need deals with the existence of extremal fixed points for nondecreasing operators defined in the space of absolutely continuous functions.

Lemma 4.2 ([8], Proposition 1.4.4) Let $I \subset \mathbb{R}$ be a nonempty closed interval and $[\alpha, \beta]$ be a nonempty functional interval in $A C(I)$. Assume that $G:[\alpha, \beta] \longrightarrow[\alpha, \beta]$ is a nondecreasing mapping and that there exists $\psi \in L^{1}(I,[0,+\infty))$ such that

$$
\left|(G \gamma)^{\prime}(x)\right| \leq \psi(x) \quad \text { for all } \gamma \in[\alpha, \beta] \text { and almost all } x \in I \text {. }
$$

Then $G$ has in $[\alpha, \beta]$ the greatest, $V^{*}$, and the least, $V_{*}$, fixed points. Moreover, they satisfy

$$
V_{*}=\min \{V: G V \leq V\}, \quad V^{*}=\max \{V: V \leq G V\} .
$$

Now we establish a new result on the existence of extremal convex solutions for problem (8).

Theorem 4.1 Assume that there exist $\alpha, \beta \in A C([c, d])$ which are, respectively, a lower and an upper solution for problem (8) satisfying $\alpha(x) \leq \beta(x)$ for all $x \in[c, d]$. Put

$$
\begin{aligned}
& {[\alpha, \beta]=\{\gamma \in A C([c, d]): \alpha(x) \leq \gamma(x) \leq \beta(x) \text { for all } x \in[c, d]\}} \\
& E=\left\{y \in \mathbb{R}: \min _{x \in[c, d]} \alpha(x) \leq y \leq \max _{x \in[c, d]} \beta(x)\right\}
\end{aligned}
$$

and assume, moreover, that the following conditions hold: 
$\left(\mathrm{H}_{1}\right)$ For all $\gamma \in[\alpha, \beta]$ and all $y \in E$, we have

$$
\liminf _{z \rightarrow y^{-}} B_{i}(z, \gamma) \geq B_{i}(y, \gamma) \geq \limsup _{z \rightarrow y^{+}} B_{i}(z, \gamma) \quad(i=1,2)
$$

$\left(\mathrm{H}_{2}\right)$ For all $y \in E$, the functions $B_{i}(y, \cdot)$ are nonincreasing in $[\alpha, \beta](i=1,2)$, that is, if $\gamma_{1}, \gamma_{2} \in$ $[\alpha, \beta]$ are such that $\gamma_{1}(x) \leq \gamma_{2}(x)$ for all $x \in[c, d]$, then $B_{i}\left(y, \gamma_{1}\right) \geq B_{i}\left(y, \gamma_{2}\right)$.

In these conditions, problem (8) has the extremal convex solutions in $[\alpha, \beta]$.

Proof We define a mapping $G:[\alpha, \beta] \longrightarrow[\alpha, \beta]$ as follows: for all $\gamma \in[\alpha, \beta], G \gamma$ is the greatest convex solution in $[\alpha, \beta]$ for the Dirichlet problem

$$
\left\{\begin{array}{l}
V^{\prime \prime}(x)+H\left(x, V(x), V^{\prime}(x)\right)=0 \quad \text { for all } x \in[c, d] \\
V(c)=\gamma_{c}, \quad V(d)=\gamma_{d}
\end{array}\right.
$$

where $\gamma_{c}, \gamma_{d}$ are the greatest solutions in, respectively, $[\alpha(c), \beta(c)]$ and $[\alpha(d), \beta(d)]$, for the following respective algebraic equations:

$$
\begin{aligned}
& B_{1}(y, \gamma)=0, \\
& B_{2}(y, \gamma)=0 .
\end{aligned}
$$

Step 1: The mapping $G$ is well defined. As $\alpha$ and $\beta$ are lower and upper solutions of (8) and by condition $\left(\mathrm{H}_{2}\right)$, we have for all $\gamma \in[\alpha, \beta]$ :

$$
\begin{aligned}
& B_{1}(\alpha(c), \gamma) \leq B_{1}(\alpha(c), \alpha) \leq 0 \leq B_{1}(\beta(c), \beta) \leq B_{1}(\beta(c), \gamma), \\
& B_{2}(\alpha(d), \gamma) \leq B_{2}(\alpha(d), \alpha) \leq 0 \leq B_{2}(\beta(d), \beta) \leq B_{2}(\beta(d), \gamma),
\end{aligned}
$$

and so condition $\left(\mathrm{H}_{1}\right)$ implies that the numbers $\gamma_{c}$ and $\gamma_{d}$ are well defined by application of Lemma 4.1.

On the other hand, as $\gamma_{c}$ and $\gamma_{d}$ are the greatest solutions of equations (21)-(22) in, respectively, $[\alpha(c), \beta(c)]$ and $[\alpha(d), \beta(d)]$, this implies that $\alpha(c) \leq \gamma_{c}, \alpha(d) \leq \gamma_{d}, \beta(c) \geq \gamma_{c}$ and $\beta(d) \geq \gamma_{d}$. So, $\alpha$ and $\beta$ are, respectively, a lower and an upper solution for problem (20). This guarantees that (20) has the greatest convex solution in $[\alpha, \beta]$. (Notice that (16)(17) provides a Nagumo-type bound for $H$ between our $\alpha$ and $\beta$.)

Step 2: $G$ is a nondecreasing mapping. Let $\gamma_{1}, \gamma_{2} \in[\alpha, \beta]$ such that $\gamma_{1}(x) \leq \gamma_{2}(x)$ for all $x \in[c, d]$, and we will show that $G \gamma_{1} \leq G \gamma_{2}$. First, notice that

$$
B_{1}\left(G \gamma_{1}(c), \gamma_{2}\right) \leq B_{1}\left(G \gamma_{1}(c), \gamma_{1}\right)=0
$$

and

$$
B_{1}\left(\beta(c), \gamma_{2}\right) \geq B_{1}(\beta(c), \beta) \geq 0
$$

so reasoning as above we obtain that $G \gamma_{1}(c) \leq G \gamma_{2}(c)$. (Note that $G \gamma_{1}(c)>G \gamma_{2}(c)$ coupled with (23)-(24) would contradict the fact that $G \gamma_{2}(c)$ is the greatest solution of $B_{1}\left(\cdot, \gamma_{2}\right)$ in 
$[\alpha(c), \beta(c)]$.) In a similar way we prove that $G \gamma_{1}(d) \leq G \gamma_{2}(d)$. Now, assume that $G \gamma_{1} \nless G \gamma_{2}$ and consider the function

$$
\hat{\alpha}(x)=\max \left\{G \gamma_{1}(x), G \gamma_{2}(x)\right\} .
$$

Thus defined, $\hat{\alpha}$ is a lower solution for problem (20) with conditions $V(c)=\gamma_{2 c}, V(d)=\gamma_{2 d}$. Indeed, if $G \gamma_{1}<G \gamma_{2}$ in an interval $\left(\hat{x}_{1}, \hat{x}_{2}\right)$, then

$$
\hat{\alpha}^{\prime \prime}(x)=G \gamma_{2}^{\prime \prime}(x)=-H\left(x, G \gamma_{2}(x),\left(G \gamma_{2}\right)^{\prime}(x)\right)=-H\left(x, \hat{\alpha}(x), \hat{\alpha}^{\prime}(x)\right) \quad \text { for all } x \in\left(\hat{x}_{1}, \hat{x}_{2}\right) .
$$

In the same way, if $G \gamma_{1}>G \gamma_{2}$ in an interval $\left(\tilde{x}_{1}, \tilde{x}_{2}\right)$, then

$$
\hat{\alpha}^{\prime \prime}(x)=-H\left(x, \hat{\alpha}(x), \hat{\alpha}^{\prime}(x)\right) \quad \text { for all } x \in\left(\tilde{x}_{1}, \tilde{x}_{2}\right) .
$$

On the other hand, if $G \gamma_{1}\left(x_{0}\right)=G \gamma_{2}\left(x_{0}\right)$, then the convexity of $G \gamma_{1}$ and $G \gamma_{2}$ implies that $D_{-} \hat{\alpha}\left(x_{0}\right)<D^{+} \hat{\alpha}\left(x_{0}\right)$.

So, problem (20) with conditions $V(c)=\gamma_{2 c}, V(d)=\gamma_{2 d}$ has a solution in $[\hat{\alpha}, \beta]$, but this contradicts the fact that $G \gamma_{2}$ is the greatest solution for this problem in $[\alpha, \beta]$. Then we conclude that $G \gamma_{1} \leq G \gamma_{2}$, and so $G$ is a nondecreasing mapping.

Step 3: $G$ has the extremal fixed points. Let $\gamma \in[\alpha, \beta]$. For all $x \in[c, d]$, we have that

$$
(G \gamma)^{\prime}(x)=(G \gamma)^{\prime}(c)-\int_{c}^{x} H\left(s, G \gamma(s), G \gamma^{\prime}(s)\right) d s,
$$

and so

$$
\left|G \gamma^{\prime}(x)\right| \leq \max \left\{\frac{|\beta(d)-\alpha(c)|}{d-c}, \frac{|\beta(c)-\alpha(d)|}{d-c}\right\}+\int_{c}^{x}|\hat{A}+\hat{B} \sqrt{s}| d s,
$$

where $\hat{A}, \hat{B}$ are as in (16). Then, by application of Lemma 4.2, we obtain that $G$ has the extremal fixed points in $[\alpha, \beta]$, say $V^{*}, V_{*}$, which moreover satisfy (19).

Step 4: $V^{*}$ is the greatest convex solution of problem $(8)$ in $[\alpha, \beta]$. First, it is clear, as $G V^{*}=V^{*}$, that $V^{*}$ is a solution of problem (8). Now, if $V$ is another solution of (8), then we have that $V \leq G V$ and so (19) implies that $V \leq V^{*}$. So, $V^{*}$ is the greatest convex solution of problem $(8)$ in $[\alpha, \beta]$.

To obtain the least convex solution of $(8)$ in $[\alpha, \beta]$, we only have to redefine the mapping $G$ in the obvious way.

Remark 4.1 Notice that condition $\left(\mathrm{H}_{1}\right)$ is satisfied, for example, if $B_{1}(\cdot, \gamma)$ is continuous or if it has only downwards discontinuities.

Remark 4.2 The same argument used in the proof of Theorem 3.3 provides that extremal convex solutions of problem (8) are also extremal convex solutions of problem (1) provided that $\frac{V(d)}{d} \leq \frac{V(c)}{c}$.

Example 4.1 Consider problem (8) in an interval $[c, d], c>0$, with the following boundary conditions: 
$\left(B_{1}\right)$ 'The initial value of the solution is one half of its mean value on the whole interval $[c, d]$;

$\left(B_{2}\right)$ 'The final value of the solution has integer part 4'.

Previous conditions can be written in the following form:

(B $\left.B_{1}\right) B_{1}(V(c), V)=V(c)-\frac{1}{2} \frac{1}{d-c} \int_{c}^{d} V(s) d s=0 ;$

$\left(\mathrm{B}_{2}\right) B_{2}(V(d), V)=-[V(d)]+4=0$.

We will show that for $V(d) \in[4,5)$ and $d \geq 3 c, \alpha(x)=\frac{V(d)}{d} x$ and $\beta \equiv V(d)$ are, respectively, a lower and an upper solution for this problem. Indeed, we have $-[\alpha(d)]=-4$ and

$$
\alpha(c)-\frac{1}{2} \frac{1}{d-c} \int_{c}^{d} \alpha(s) d s=\frac{V(d)}{d}\left(c-\frac{d+c}{4}\right) \leq 0
$$

if $d \geq 3 c$. On the other hand, the constant function $\beta \equiv V(d)$ is such that

$$
B_{1}(\beta(c), \beta)=\frac{1}{2} V(d) \geq 0
$$

and

$$
B_{2}(\beta(d), \beta)=-[V(d)]+4=0 .
$$

Finally, notice that for each $V(d) \in[4,5)$, functions $B_{1}$ and $B_{2}$ satisfy conditions $\left(\mathrm{H}_{1}\right)-\left(\mathrm{H}_{2}\right)$ between $\alpha$ and $\beta$. We can conclude, by application of Theorem 4.1, that if $d \geq 3 c$ then problem (8) with boundary conditions $\left(\mathrm{B}_{1}\right)-\left(\mathrm{B}_{2}\right)$ has the extremal solutions between $\alpha$ and $\beta$ for each $V(d) \in[4,5)$.

Remark 4.3 In papers [3] and [1] the authors obtained uniqueness of solutions for problem (2). Notice that uniqueness of solutions cannot be guaranteed when we include variable coefficients $p$ and $q$ and functional boundary conditions. For example, consider problem (1) with boundary conditions

$$
[V(1)]=2, \quad[V(2)]=4
$$

In this case, each function $V(x)=a x$ with $a \in[2,2.5)$ is a solution of the problem.

Competing interests

The authors declare that they have no competing interests.

Authors' contributions

The two authors contributed in the same way in the development of this paper. All authors read and approved the final manuscript.

\section{Author details}

${ }^{1}$ Department of Mathematical Analysis, University of Santiago de Compostela, Campus Vida, Santiago de Compostela, 15782, Spain. '2Department of Mathematics, CEMAPRE - ISEG, University of Lisboa, Rúa do Quelhas, 6, Lisboa, 1200-781, Portugal.

\section{Acknowledgements}

The first author was partially supported by Xunta de Galicia, Consellería de Cultura, Educación e Ordenación Universitaria, through the project EM2014/032 'Ecuacións diferenciais non lineares'; and by Ministerio de Economía y Competitividad of Spain under Grant MTM2010-15314, cofinanced by the European Community fund FEDER. The second author was 
partially funded by Fundação para a Ciência e Tecnologia through the project UID/Multi/00491/2013 and the Transnational Cooperation FCT Portugal-Slovakia 'Analysis of Nonlinear Partial Differential Equations in Mathematical Finance (2013-2014)' and by the EU Grant Program FP7-PEOPLE-2012-ITN STRIKE - 'Novel Methods in Computational Finance', No. 304617 (D.S.).

Received: 5 June 2015 Accepted: 10 August 2015 Published online: 22 August 2015

References

1. Grossinho, MR, Morais, E: A fully nonlinear problem arising in financial modelling. Bound. Value Probl. 2013, 146 (2013)

2. Black, F, Scholes, M: The pricing of options and corporate liabilities. J. Polit. Econ. 81, 637-654 (1973)

3. Amster, P, Aberbuj, CG, Mariani, MC, Rial, D: A Black-Scholes option pricing model with transaction costs. J. Math. Anal. Appl. 303, 685-695 (2005)

4. Grossinho, MR, Morais, E: A note on a stationary problem for a Black-Scholes equation with transaction costs. Int. J. Pure Appl. Math. 51, 579-587 (2009)

5. De Coster, C, Habets, P: Two-Point Boundary Value Problems: Lower and Upper Solutions. Mathematics in Science and Engineering, vol. 205. Elsevier, Amsterdam (2006)

6. Cherpion, M, De Coster, C, Habets, P: Monotone iterative methods for boundary value problems. Differ. Integral Equ. 12(3), 309-338 (1999)

7. Franco, D, Pouso, RL: Nonresonance conditions and extremal solutions for first-order impulsive problems under weak assumptions. ANZIAM J. 44(3), 393-407 (2003)

8. Heikkilä, S, Lakshmikantham, V: Monotone Iterative Techniques for Discontinuous Nonlinear Differential Equations Dekker, New York (1994)

\section{Submit your manuscript to a SpringerOpen ${ }^{\circ}$ journal and benefit from:}

- Convenient online submission

Rigorous peer review

- Immediate publication on acceptance

Open access: articles freely available online

- High visibility within the field

- Retaining the copyright to your article 